\title{
MIXING-MODEL SENSITIVITY TO INPUT PARAMETER VARIATION
}

\author{
JOSIAH BIGELOW ${ }^{1,2}$, HUMBERTO SILVA III $^{1,2}$, C. RANDALL TRUMAN ${ }^{2} \&$ PETER VOROBIEFF $^{2}$ \\ ${ }^{1}$ Sandra National Laboratories, Albuquerque, USA \\ ${ }^{2}$ Department of Mechanical Engineering, The University of New Mexico, USA
}

\begin{abstract}
Amagat and Dalton mixing-models were analyzed to compare their thermodynamic prediction of shock states. Numerical simulations utilized the Sandra National Laboratories (SNL) shock hydrodynamic code CTH [1]. Simulations modeled the University of New Mexico (UNM) shock tube laboratory experimental series shocking a 1:1 molar mixture of helium $(\mathrm{He})$ and sulfur hexafluoride $\left(\mathrm{SF}_{6}\right)$. Five input parameters were varied for sensitivity analysis: driver section pressure; driver section density; test section pressure; test section density; and mixture ratio (mole fraction). We show via incremental Latin hypercube sampling (LHS) analysis that significant differences exist between Amagat and Dalton mixing-model predictions. The differences observed in predicted shock speeds, temperatures, and pressures grow more pronounced with higher shock speeds.
\end{abstract}

Keywords: Amagat, Dalton, verification, validation, incremental Latin hypercube sampling, shock tube.

\section{INTRODUCTION}

When considering how to numerically model a mixture of gases, the following question arises: what model is most accurate at predicting the thermodynamic properties of the mixture at a given temperature and pressure? Numerous papers have been published about multiphase and multi-component flows [2], reacting flows, flows with arbitrary equations of state [3], and mixtures at relatively low pressures [4]. Furthermore, papers have been published on the behavior of the shock region using Monte Carlo methods in binary gas mixtures of perfect gases [5, 6]. However, one cannot make rigorous convergence assertions with Monte Carlo methods. Beattie [7, 8] discusses mixtures of real gases. However, there is very little available on which model (Amagat or Dalton) best applies to the case of a mixture of nonreacting, real gases, undergoing shock conditions. One may assume from the context of the available material that Amagat's Law would be the natural choice but it is not entirely clear that this is true $[2,9,10,11]$. In this paper we explore Amagat and Dalton predictions of shock speed, pressure, and temperature for a 1:1 molar mixture of $\mathrm{He}$ and $\mathrm{SF}_{6}$. This is accomplished via numerical simulations of a shock tube utilizing CTH and Latin hypercube sample (LHS) analysis.

\section{THEORY}

The problem of how to model mixtures of real gases presents the question of which of the three measurable properties, pressure $(P)$, temperature $(T)$, and volume $(V)$, apply to the whole mixture. Often the assumption is made that the measured temperature applies to the mixture leaving $P$ and $V$ as the variables that are a function of the components [9]. We know that for a pure, real gas the following relationship holds [10]

$$
P V=m R_{g} T z,
$$


where $z=z(P, T)$ is the compressibility factor of the gas, $m$ is the mass, and $R_{g}$ is the specific gas constant. From Amagat, we know that

$$
V=\sum_{i=1}^{N} V_{i}
$$

where $V_{i}$ is the volume occupied by component $i$ at the given temperature and pressure of the mixture, otherwise known as the partial volume relation [9]. On the other hand, from Dalton we find that

$$
P=\sum_{i=1}^{N} P_{i},
$$

where $P_{i}$ is the pressure exerted by the $i$ th component at the given temperature and volume of the mixture, or the partial pressure relation [9]. For ideal pure gases $(z=1)$ Amagat's and Dalton's law give the same results [9]. Thus a non-ideal gas or non-ideal set of conditions must be imposed upon our mixture. Shocks are non-isentropic processes that allow us to study gases in a non-ideal state.

\subsection{Numerical analysis}

Shock modeling is inherently difficult in a numerical sense [1]. Great care is required to make codes stable and accurate across the shock since a shock is often represented as a discontinuous jump state, introducing a cusp into the numerical results often leading to numerical instability [1]. This research is interested in differences that may be present in mixing formulations but not what is happening inside of the shock. The primary interest is in the effect of the shock front as it passes through the gas mixture. Any numerical algorithm utilized need only accurately represent the shock speed, shock pressure, and shock temperature, but does not need to provide an accurate representation of the shock thickness or interior structure.

\subsection{Verification and validation}

Verification and validation are distinct and important aspects of any numerical analysis. Verification comprises, but is not limited to the following: have input decks been found to be free of typographical errors, was the mathematical analysis performed correctly, how does the chosen mesh affect the model solution, etc.? Verification is an objective measure since there are clear correct and incorrect implementations of a representative physical algorithm(s). On the other hand, validation is harder to achieve because it speaks to the usefulness of the algorithm(s) as applied. Assumptions, numerical metrics of comparison, algorithm choice, etc. all play into the validity of observed numerical results. Validation requires carefully analyzing the results which should include a sensitivity analysis of the quantities of interest (QoI) in order to make rigorous validity conclusions. Generating a validation assertion requires objective as well as subjective judgment, statistics, and properly chosen validity metrics. For this research codes were used that have been validated for various shock states and equations of state (EOS) applications [1, 12]. To that effect, RankineHugoniot analysis provided that EOS surfaces passed through the correct thermodynamic states. Future validation will come through comparison to experimental values and exercising models via incremental LHS analysis. 


\subsection{Incremental Latin hypercube sampling}

Incremental samples mean that no sample value is ever reused when you increase sample size. This allows a convergence statement to be made about the results since values are going to begin to fill in the entire study space as more samples are run. The term Latin Hypercube derives from a $k$-dimensional extension of Latin Square sampling [13]. A Latin Square is a sparse matrix where any given row and column contains only one value. Wyss and Jorgensen show an excellent example of a Latin Square in the DAKOTA User Guide [14]. Probability bins (most often in a cumulative distribution function) are successively subdivided in an incremental sampling ensuring that the tails of a distribution are accurately represented. This feature of an incremental LHS study is the second part of convergence. As more samples are run in a simulation from the LHS input 'stack', systematic variation in the inputs forces systematic variation in the output across the entire PDF. Convergence is determined by measuring the linear correlation strength of input variables to output variables pairwise. When correlation order and then correlation strength ceases to change, the incremental LHS study has converged, which is a strict convergence criteria.

\subsection{Equation of State (EOS)}

\section{METHODOLOGY}

The Jacobs-Cowperthwaite-Zwisler (JCZ) EOS was originally developed to give a general representation over a wide range of material densities [15]. SNL updated the original TIGER [15] code and JCZ EOS with improved fits over ionization ranges and additional molecular species in what is now called the JCZS EOS $[12,16]$. Hobbs et al. [12] updated the original Grüneisen function to depend on a molecular potential function, EXP6, which is similar in form to the Lennard-Jones (LJ) potential [12]. Thus, the Grüneisen function in the JCZS EOS

$$
P=\frac{G(V, T, \phi) n R T}{V}+P_{0}(V, \phi)
$$

is based on the EXP6 function

$$
\phi(r)=\epsilon\left[\left(\frac{6}{\eta-6}\right) e^{\eta\left(1-r / r^{*}\right)}-\left(\frac{\eta}{\eta-6}\right)\left(\frac{r^{*}}{r}\right)^{6}\right] .
$$

Variable $\eta$ is a fit parameter which has been shown to affect the EXP6 potential relatively little $[12,16]$. Due to the insensitivity of the EXP6 potential to $\eta$, most implementations use $\eta \approx 13$ [12]. The parameters $r^{*}$ and $\epsilon$ are the molecular distance of separation at the minimum potential energy and well depth for the pair potential, respectively. The variable $r$ is the distance by which the molecules are separated. The advantage of $\epsilon$ and $r^{*}$ is that they can be calculated accurately from generally well-characterized quantities, such as the heat of formation, molecular number, and composition. Having only two parameters to calculate makes the JCZS EOS ideal for use on a wide variety of gases [12]. Numerical analogues to the JCZS EOS were implemented in the form of SESAME tables.

The SESAME format of tabular EOS, developed by Los Alamos National Laboratory (LANL), is a flexible EOS format allowing custom table generation [17]. The TIGER code set was utilized to generate the SESAME tables for the simulations by computing equilibrium thermodynamic states over a range of temperatures and volumes representing tabular EOS for Amagat or Dalton mixtures. Construction of a mixed EOS was performed by taking outputs of a TIGER data run from each pure gas and summing over either the volumes (Amagat) or 
the pressures (Dalton) from each pure gas to create a single table. Two nuances to this process are worth mentioning. Firstly, eqn (2) for the Amagat model is presented with volume as an extensive parameter. When summing specific volumes (an intensive parameter) sums must be weighted by mass fraction

$$
v(T, P)=\sum_{i} w_{i} v_{i}(T, P),
$$

where $w_{i}=m_{i} / m_{\text {tot }}$ is the $i$ th mass fraction, $m_{i}$ is the $i$ th mass, and $m_{t o t}$ is the mixture total mass. Similarly, specific energy in TIGER is represented as an intensive parameter with respect to mass and is most easily summed in a mass weighted-fashion. Secondly, since specific volume is an intensive parameter, a scaling must be performed for the Dalton table. Given that the temperature and volume are the independent parameters in a Dalton mixing scheme, equivalence of state is required to get an accurate representation of the mixed pressure state. For equivalence to occur, the volumes must be equalized. Recall that

$$
V=m v=M n v,
$$

where $M$ is molecular mass. In the case of Dalton, we are considering partial pressure of each constituent at the given temperature and volume. In other words, $P_{i}=P(T, V)$. However, we have $P_{i}=P\left(T, v_{i}\right)$, which is equivalent, but not identical in the sense that $V \neq v$. In order to sum over the He and $\mathrm{SF}_{6}$ pressures for a Dalton formulation, we must demand that both pure $\mathrm{He}$ and $\mathrm{SF}_{6}$ tables represent the same $(T, v)$ states. The way to achieve this is to scale the specific volume of either the $\mathrm{He}_{\text {or }} \mathrm{SF}_{6}$. Taking eqn (7) we can formulate a scaling factor for He volumes

$$
m_{H e} v_{H e}=V=m_{S F_{6}} v_{S F_{6}} \Rightarrow v_{H e}=v_{S F_{6}} \frac{m_{S F_{6}}}{m_{H e}} .
$$

The process used was to generate $\mathrm{SF}_{6}$ states in TIGER and use $T$ and scaled $v$ values to generate the He states. The column of volume values for the He input was scaled as shown in eqn (8). A simple summation of the computed He pressures with the $\mathrm{SF}_{6}$ pressure values at each $(T, v)$ state was then performed.

Refinements to the SESAME table were handled on the basis of levels. A level describes how small $\Delta T, \Delta v$, or $\Delta P$ are. Refinement means to reduce the size of the step between temperatures, volumes, or pressures. The first level is the coarsest level with each level above it representing a halving of $\Delta X ; X$ being the thermodynamic state variable of interest. Since $X$ represents temperature and then volume, respectively, the density of points on the $(T, v)$ surface would increase by a factor of four, for a 2D problem, with every subsequent refinement level. From ideal gas calculations the maximum shock temperature was found to be around $850 \mathrm{~K}$. A SESAME table was generated with TIGER performing the mixing to estimate mixture deviation from ideal behavior and help set bounds on $T, v$, and $P$. The pure $\mathrm{He}$ and $\mathrm{SF}_{6}$ tables were generated for both the Amagat and Dalton mixtures. In order to provide some bound that could account for higher pressures or temperatures, $T_{\max }=1500 \mathrm{~K}$ was selected. Correspondingly $T_{\min }=180 \mathrm{~K}$ was chosen for the lower temperature bound. Similarly $v_{\max }=6005 \mathrm{cc} / \mathrm{g}$ and $v_{\min }=5 \mathrm{cc} / \mathrm{g}$ were chosen for the mixture. The initial values of $\Delta T$ and $\Delta v$ were picked to provide relatively coarse spacing without being so large that many refinement levels were required for convergence. The maximum and minimum values of each property were held fixed to provide the proper refinement sequence. Table 1 shows the details of the first three refinements for the SESAME files. A convergence study was performed on the SESAME tables by holding the initial conditions and spatial mesh constant in 12 different shock tube configurations studied by Trueba Monje and Yoo [11]. 
Table 1: SESAME refinement levels.

\begin{tabular}{ccccc}
\hline Level & $N_{T}$ & $\Delta T[K]$ & $N_{v}$ & $\Delta v\left[\frac{c c}{g}\right]$ \\
\hline 1 & 56 & 24 & 121 & 50 \\
2 & 112 & 11.892 & 242 & 24.896 \\
3 & 224 & 5.919 & 484 & 12.422 \\
\hline
\end{tabular}

Table 2: Driver and test section pressures [11].

\begin{tabular}{cc}
\hline Driver pressure (psia) & Test pressure (psia) \\
\hline 146 & 1.14 \\
166 & 5.70 \\
186 & 11.4 \\
- & 17.1 \\
\hline
\end{tabular}

One of the driver pressures and one of the test section pressures pairwise in Table 2 defines each of the 12 cases. Shock speeds were compared via absolute relative error of the shock speeds between the different levels to assess change due to the SESAME refinement. A relative error less than $1 \times 10^{-3}$ was determined to be sufficiently refined. Results showed that three refinement levels were sufficient to accurately model shock behavior.

\section{$3.2 \mathrm{CTH}$}

CTH was selected because it is a hydrodynamics code that is written to study shock physics with the ability to input tabular EOS, allowing for the study of variations in customized EOS formulations [1]. The CTH solution scheme is a two-step Eulerian method where the mesh first deforms with the material in a Lagrangian sense and then is mapped back to the original Eulerian positions [1]. There are six mesh options in CTH: 1D rectangular, cylindrical, and spherical coordinates, 2D rectangular and cylindrical, and 3D rectangular coordinates [1]. CTH implements the SESAME tabular EOS format in the form of binary files [1, 17].

$\mathrm{CTH}$ models were developed to simulate experiments performed in the shock tube at the University of New Mexico (UNM) with a mixture of $\mathrm{He}_{\text {and }} \mathrm{SF}_{6}$ [11]. The availability of this experimental data allows the numerical simulations to be validated. An input deck was written to capture the geometry of the UNM shock tube in both 1D and 2D configurations. Limited preliminary simulations were run in $3 \mathrm{D}$ and the results more closely matched the experimental results. However, 3D simulations did not change the trends observed between Amagat or Dalton and would not have changed any conclusions drawn from the 2D simulations. Simulations in 3D take significantly longer to run and are currently being processes for an incremental LHS analysis. Furthermore, since the trends did not change between 2D and 3D, a suite of 3D simulations was deemed unnecessary for an LHS study. A schematic image of the shock tube is provided with dimensions in Fig. 1.

The UNM shock tube is a $7.62 \mathrm{~cm}$ ID round aluminum tube coupled to a $7.62 \mathrm{~cm}$ square steel tube. The round tube is the high pressure or driver section, while the square tube comprises the low pressure or test section. The driver section is separated from the test section via a polypropylene membrane, which is punctured by a pneumatically-driven broadhead 


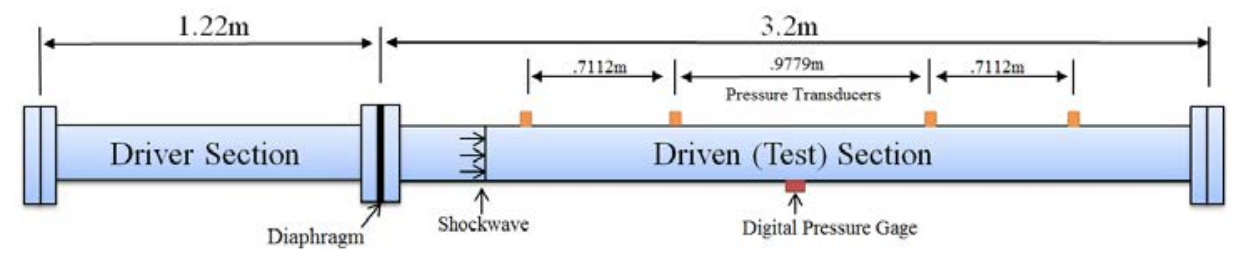

Figure 1: Notional depiction of UNM shock tube [11].

arrow head on the axis of the driver section [11]. When the membrane is punctured the gas flow due to pressure imbalance produces a traveling planar shock wave. The test section can be easily modified to allow for pressure transducers, thermocouples, viewing windows, etc. due to its square shape (the sides are flat).

The shock wave produces a pressure and temperature spike as it passes through the test section [18]. Precise spacing of the pressure transducers allows for measurement of both the shock over-pressure and shock speed. The numerical model captures the center-line of the tube in the transverse direction in the 2D and 1D cases; thus the transition from round to square tubing is not modeled. The pressure transducers are replicated by tracers in CTH at the locations of the pressure transducers in the experiment allowing for a replication of the measurements made in the experiments. The membrane was not modeled, but assumed to vanish when it ruptured. Thus gases in the driver and test sections on either side at their respective pressures and densities were placed in contact at rupture. Just as in the experiment, in the simulation a shock was formed by the high-pressure gas moving into the low-pressure $\mathrm{He}_{-} \mathrm{SF}_{6}$ mixture. The walls of the tube were implemented via a reflecting boundary condition on 2,4 , or 6 sides depending on whether the simulation was $1 \mathrm{D}, 2 \mathrm{D}$, or $3 \mathrm{D}$ respectively.

\subsection{Mesh convergence via refinement analysis}

Convergence with refinement of the spatial grid was also studied. The shock tube is a $7.62 \mathrm{~cm}$ ID $\times 122 \mathrm{~cm}$ (driver) section coupled to a $7.62 \mathrm{~cm}$ square $\times 320 \mathrm{~cm}$ (test) section for an overall length of $442 \mathrm{~cm}$. For level 1 the computational grid was 8 by 406 nodes, which gives a spacing of $\Delta x \approx \Delta y \approx 1.09 \mathrm{~cm}$. The grid size refinements progressed by doubling the number of nodes in each direction. Table 3 shows the spatial refinement for the first five levels and corresponding step sizes. Methods detailed in Roache were used to estimate the convergence of the solution with mesh refinement [19]. Modeling the tube with a level 3 refinement took considerably longer to run but reduced the estimated error only slightly. A level 4 refinement was not run but a level 5 refinement was performed for all cases as a check. This was important because while CTH uses second-order-accurate methods for advection

Table 3: Spatial refinement levels.

\begin{tabular}{ccccc}
\hline Level & $N_{h}$ & $\Delta y[\mathrm{~cm}]$ & $N_{L}$ & $\Delta x[\mathrm{~cm}]$ \\
\hline 1 & 8 & 1.089 & 406 & 1.091 \\
2 & 16 & 0.508 & 812 & 0.545 \\
3 & 32 & 0.2458 & 1624 & 0.2723 \\
4 & 64 & 0.1210 & 3248 & 0.1361 \\
5 & 128 & 0.0600 & 6496 & 0.0681 \\
\hline
\end{tabular}


and mesh remap, results are not necessarily second-order-accurate since some numerical dissipation is present due to adjustments to the first and second coefficients of viscosity required for numerical stability across the shock [1]. In some sense, the same simulation is not run twice since the value of the viscosities change based on simulation parameters. In other words, convergence cannot occur in a Richardson sense where error decreases as $\mathcal{O}\left(h^{p}\right)$ with refined mesh size. We found that the relative errors between a level 1, 2, and 3 mesh simulations were $\leq 10^{-3}$ for shock speed, shock pressure, and temperature. Furthermore, level 5 mesh results did not change from the level 3 results, giving confidence in simulation convergence. For aforementioned reasons we used the baseline mesh for all LHS studies.

\subsection{LHS}

The SNL software package DAKOTA was used to generate pseudo-random samples from probability distributions describing input variables used for the LHS analysis. Starting with 125 samples, simulations were run, doubling sample size every at each succeeding refinement, which is a sampling restriction for incremental LHS. When the correlation magnitude of input variable to shock speed, pressure, and temperature stopped changing in order and value, the simulation was determined to have converged. The driver and test sections' pressures and densities were varied along with the mass fraction of helium in the test section mixture. Parameter definitions are shown in Table 4 and the resulting shock pressures, temperatures, and speeds were compared for different values of variables $\chi_{1}$ through $\chi_{5}$ to determine sensitivity as well as differences between the predictions using Amagat and Dalton models.

The same case was run with both Amagat and Dalton tables so that they could be directly compared. Parameter distributions were selected based on an analysis of the experimental inputs and fitting distributions (normal distributions were bounded at $\pm 3.08 \sigma$ ). The decision was made to model just one of the 12 driver and test pressure combinations for the LHS study. Driver pressure $166 \mathrm{psia}\left(1.145 \times 10^{7} \mathrm{dyne} / \mathrm{cm}^{2}\right)$ and test section pressure $11.4 \mathrm{psia}$ $\left(7.860 \times 10^{5}\right.$ dyne $\left./ \mathrm{cm}^{2}\right)$ were selected. This pressure pair struck a balance between shock speed (faster with lower test section pressures) and shock pressure (higher with higher test section pressures). Since the goal is to examine the sensitivity of shock tube simulations to variations in the independent variables rather than forming a comprehensive response surface, it was deemed acceptable to model only one case in the middle of driver and test section pressures. Tables 5 and 6 list variable parameters used for the normal and triangular distributions, respectively. Distributions were selected based off of minimizing the negative $\log$ likelihood function. However, $\chi_{2}, \chi_{3}$, and $\chi_{4}$ exhibited small variation from the experimental data and were fit with a triangular distribution based off of comparable variation observed in similar variables.

Table 4: Parameters to be perturbed.

\begin{tabular}{lcc}
\hline Parameter & Symbol & Units \\
\hline Driver pressure & $\chi_{1}$ & {$\left[\mathrm{dyne} / \mathrm{cm}^{2}\right]$} \\
Test pressure & $\chi_{2}$ & {$\left[\mathrm{dyne} / \mathrm{cm}^{2}\right]$} \\
Driver density & $\chi_{3}$ & {$\left[\mathrm{~g} / \mathrm{cm}^{3}\right]$} \\
Test density & $\chi_{4}$ & {$\left[\mathrm{~g} / \mathrm{cm}^{3}\right]$} \\
Helium mass fraction & $\chi_{5}$ & {$[$ number $]$} \\
\hline
\end{tabular}


Table 5: Normal distribution fits.

\begin{tabular}{ccccc}
\hline Parameter & $\mu$ & $\sigma$ & Low bound & High bound \\
\hline$\chi_{1}$ & $11.4471 \times 10^{6}$ & $13.283 \times 10^{3}$ & $11.4062 \times 10^{6}$ & $11.4880 \times 10^{6}$ \\
$\chi_{5}$ & 0.0267 & 0.0033 & 0.0165 & 0.0369 \\
\hline
\end{tabular}

Table 6: Triangular distribution fits.

\begin{tabular}{cccc}
\hline Parameter & $\mathrm{a}$ & $\mathrm{b}$ & $\mathrm{c}$ \\
\hline$\chi_{2}$ & $77.2213 \times 10^{4}$ & $79.9792 \times 10^{4}$ & $78.6002 \times 10^{4}$ \\
$\chi_{3}$ & $1.8537 \times 10^{-3}$ & $1.8780 \times 10^{-3}$ & $1.8658 \times 10^{-3}$ \\
$\chi_{4}$ & $23.1943 \times 10^{-4}$ & $27.9275 \times 10^{-4}$ & $25.5376 \times 10^{-4}$ \\
\hline
\end{tabular}

\section{RESULTS}

LHS results were analyzed in an incremental fashion using the correlation coefficient

$$
r_{x, y}=\frac{\operatorname{cov}(x, y)}{\sigma_{x} \sigma_{y}}
$$

where $r_{x y}$ is the correlation between $x$ and $y, \operatorname{cov}()$ is the covariance, and $\sigma_{i}$ is the $i$ th standard deviation. The base sample set consisted of 125 pseudorandom samples and the number of samples doubled with every 'step'. Starting with the base sample set of 125 values the magnitude of the correlation coefficient, $r$, was computed, against $U_{s}, P_{s}$, and $T_{s}$ and $\chi_{1}$ through $\chi_{5}$ in a pairwise manner for every doubling. Once the order and the magnitude of the correlation for the statistically significant variables became constant, convergence was achieved. Histograms of the correlation of the shock speeds, $U_{s}$, pressures, $P_{s}$, and temperatures, $T_{s}$, to independent variables $\chi_{1}$ through $\chi_{5}$ were plotted to determine convergence. Simulation results converged after 1000 samples (three doublets) of input variables (defined in Table 4) were run.

Fig. 2 shows correlation coefficients for the LHS study with Amagat and Dalton QoI (Quantities of Interest) in the left and right columns, respectively. It is clear that both the Amagat and Dalton models are most sensitive to $\chi_{4}$, test section density, and $\chi_{5}$, helium mass fraction. However, which variable has the strongest relationship depends on the QoI. The sensitivity of the two models is quite similar in magnitude and ordering of variables, $\chi_{i}$, based on correlation strength. Only shock pressure shows a significant difference in sensitivity between the Amagat and Dalton models in the second-most influential parameter $\chi_{5}$, helium mass fraction. We fit the predicted QoI in the LHS study with probability density functions (generalized extreme value) for both the Amagat and Dalton models. We then calculated the coefficient of overlap [20,21], $C_{\text {ovl }}$, between the two distributions and present the results in Figs 3, 4, and 5. Fig. 3 shows that there exists strong agreement between the Amagat and Dalton EOS when it comes to predicting shock speed. The overlap between the two distributions comprises $80.58 \%$ of the simulated values based on the value of $C_{\text {ovl }}$. One way of thinking about this result is that the distance between the Amagat and Dalton shock speed distributions is not large.

Similarly, shock pressure distributions in Fig. 4 show that the pressures also have strong agreement, as the overlap constitutes $75.27 \%$ of the simulated values. While slightly less than shock speed overlap, the agreement between the two distributions is still significant. 

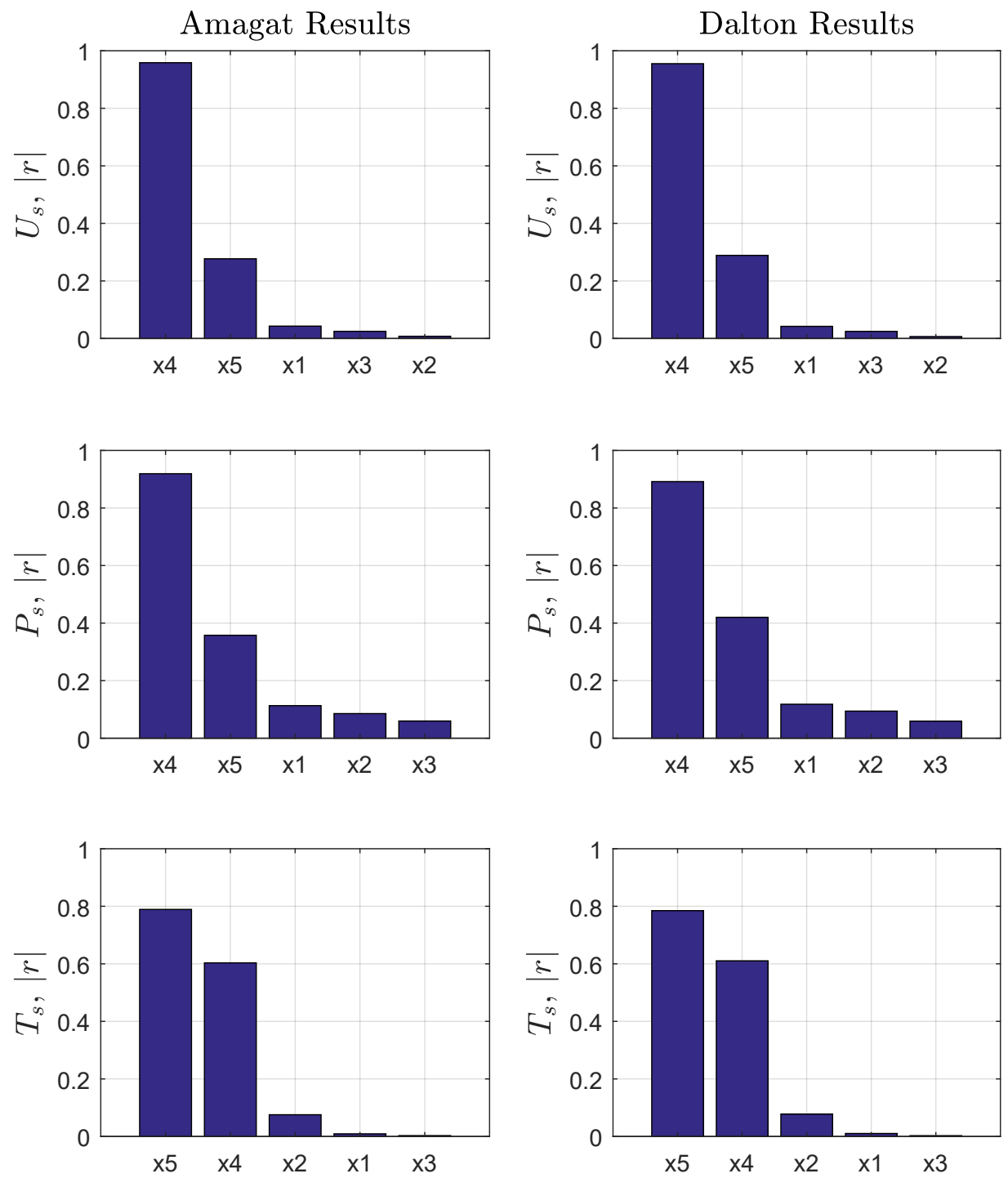

Figure 2: LHS QoI results run against 1000 samples.

The shock temperature predictions showed the greatest disparity between the two models as the overlap only accounted for $29.02 \%$ of the simulated values. Fig. 5 shows the two temperature distributions and the region of overlap. This is the largest difference between the predictions using the Amagat and Dalton models.

\section{CONCLUSIONS}

LHS results in Figs 3 and 5 show strongest agreement between Amagat and Dalton models in the shock speed and the poorest agreement in shock temperature. In addition, the LHS results showed that the shock pressure has the most complex relationship to 


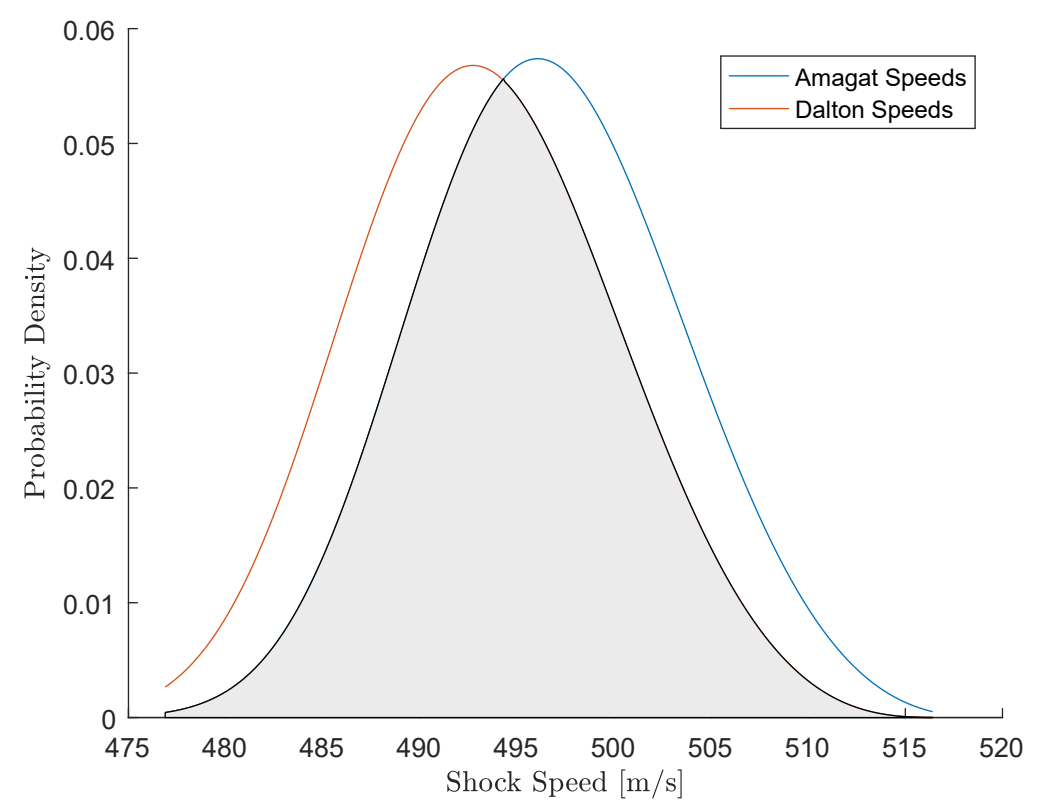

Figure 3: Overlap in shock speed distributions, $C_{\text {ovl }}=0.8058$.

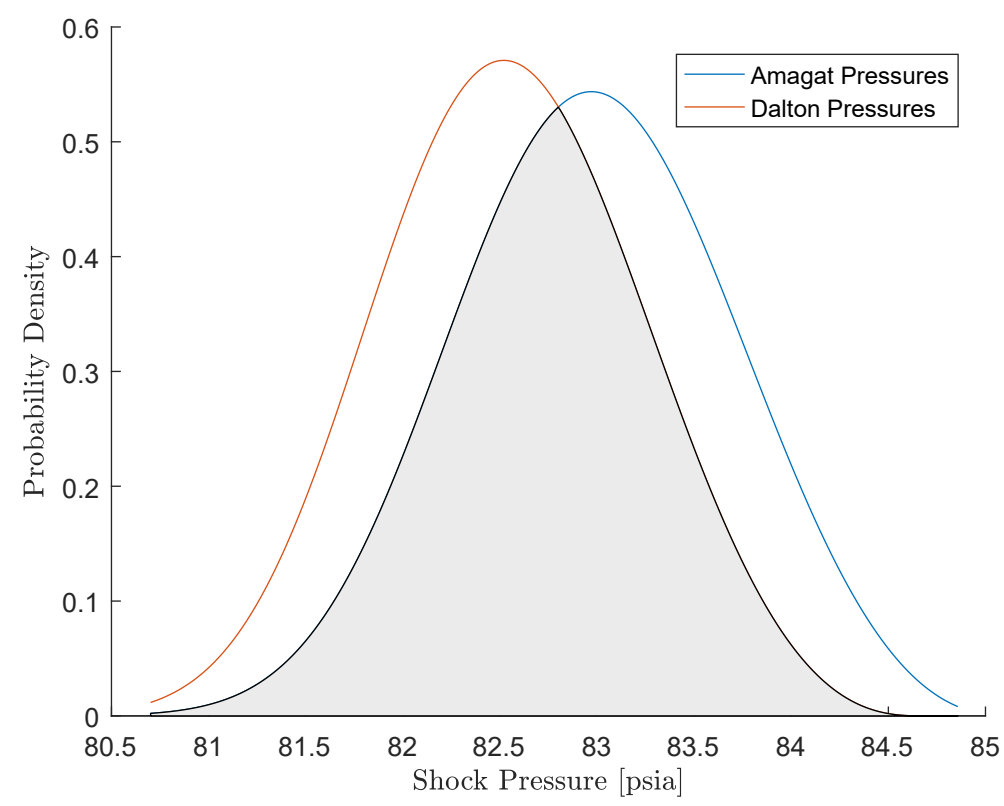

Figure 4: Overlap in shock pressure distributions, $C_{\text {ovl }}=0.7527$.

the input parameters perturbed. Shock speed and shock temperature were dominated by variables $\chi_{4}$ and $\chi_{5}$. Predominantly shock pressure, while still dependent on $\chi_{4}$ and $\chi_{5}$, has stronger relationships with $\chi_{1}, \chi_{2}$, and $\chi_{3}$ than either shock speed or shock 


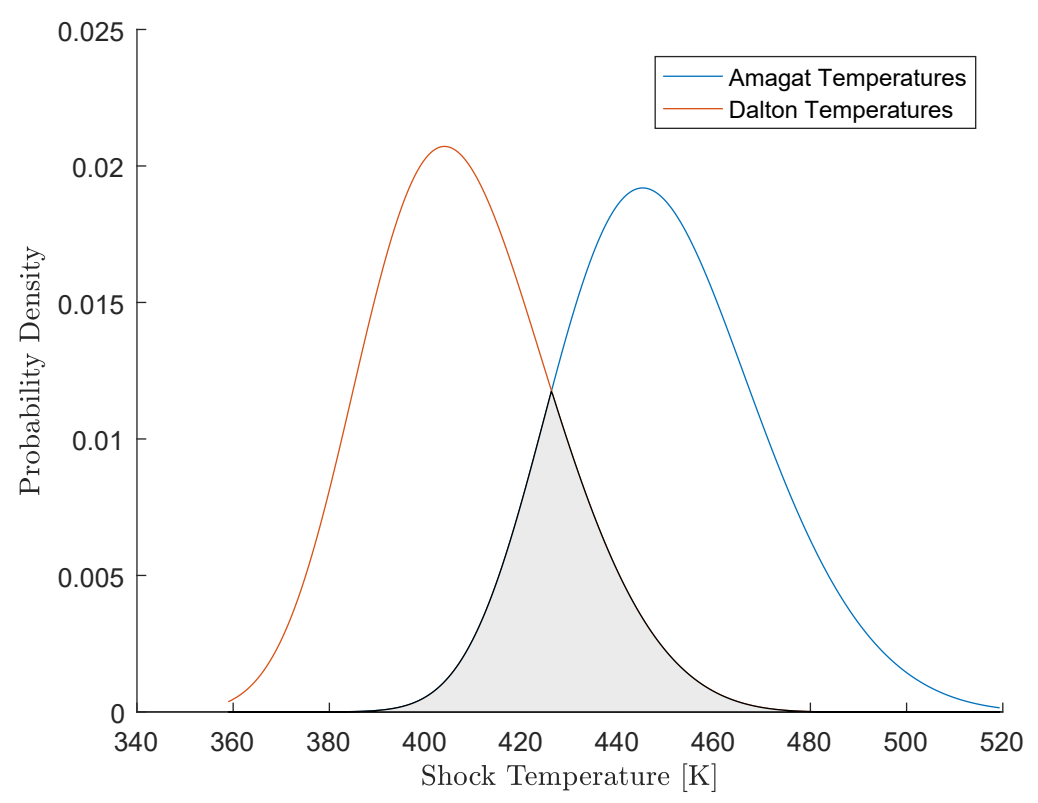

Figure 5: Overlap in shock temperature distributions, $C_{\text {ovl }}=0.2902$.

temperature do. Furthermore the LHS analysis showed that while the Amagat and Dalton EOS models were sensitive to essentially the same parameters, it also showed that they predict different shock speeds, pressures, and temperatures. As was shown in Figs 4, and 5, the models only agree well on 2 out of the 3 shock characteristics. This implies that the models cannot simultaneously agree on both pressure and temperature of the mixture. This might be expected from different treatments of energy models. When modeling gas mixtures undergoing shocks, we conclude that Amagat and Dalton mixing models produce significantly different results. We anticipate that comparison with shock tube experiments will allow us to determine which model is more accurate.

\section{REFERENCES}

[1] McGlaun, J.M., Thompson, S.L. \& Elrick, M.G., CTH: A three-dimensional shock wave physics code, International Journal of Impact Engineering, 10, pp. 351-360, 1990.

[2] Li, D., Sankaran, V., Lindau, J.W. \& Merkle, C.L., A unified computational formulation for multi-component and multi-phase flows. AIAA Paper 2005-1391, January 2005.

[3] Merkle, C.L., Sullivan, J.Y., Buelow, P.E.O. \& Sankaran, V., Computation of flows with arbitrary equations of state. AIAA Journal, 36(April), pp. 515-521, 1998.

[4] Tow, P.S., Evidence of validity of Amagat's law in determining compressibility factors for gaseous mixtures under low and moderate pressures. Journal of Physical Chemistry, 68(July), pp. 2021-2023, 1964.

[5] Bird, G.A., The structure of normal shock waves in a binary gas mixture. Journal of Fluid Mechanics, 31(4), pp. 657-668, 1968.

[6] Sherman, F.S., Shock-wave structure in binary mixtures of chemically inert perfect gases. Journal of Fluid Mechanics, 8(3), pp. 465-480, 1960. 
96 Computational and Experimental Methods in Multiphase and Complex Flow IX

[7] Beattie, J.A., The computation of the thermodynamic properties of real gases and mixtures of real gases. Symposium on Thermodyanmics and Molecular Structure of Solutions, September 1948.

[8] Beattie, J.A., A rational basis for the thermodynamic treatment of real gases and mixtures of real gases. Physical Review, 36(240), pp. 132-145, 1930.

[9] Woo, K.W. \& Yeo, S.I., Dalton's law vs. Amagat's law for the mixture of real gases. The SNU Journal of Education Research, 5, pp. 127-134, 1995.

[10] Van Wylen, G.J. \& Sonntag, R.E., Fundamentals of Classical Thermodynamics, 2nd ed, Wiley: Hoboken, NJ, pp. 405-420, 431-476, 1973.

[11] Trueba Monje, I. \& Yoo, J.H., Investigation of Dalton's law and Amagat's law for mixtures using shock wave propagation. AIAA Region IV Student Conference Paper, April 2016.

[12] Hobbs, M., Baer, M.R. \& McGee, B.C., JCZS: An intermolecular potential database for performing accurate detonation and expansion calculations. Propellants, Explosives, Pyrotechnics, 24, pp. 269-279, 1999.

[13] Mckay, M.D., Beckman, R.J. \& Conover, W.J., A comparison of three methods for selecting values of input variables in the analysis of output from a computer code. Technometrics, 42, pp. 55-61, 2000.

[14] Wyss, G.D. \& Jorgensen, K.H., A users's guide to LHS: Sandia's Latin hypercube sampling software. Software Documentation SAND98-0210, Sandia National Laboratories, 1998.

[15] Cowperthwaite, M. \& Zwisler, W., The JCZ equations of state for detonation products and their incorporation in the TIGER code. Sixth Symposium (International) on Detonation, no. ACR-221, pp. 162-172, 1976.

[16] McGee, B., Hobbs, M. \& Baer, M., Exponential 6 parametrization for the JCZ3-EOS. Sandia Report SAND98-1191, Sandia National Laboratories, 1998.

[17] Lyon, S. \& Johnson, J., SESAME: The Los Alamos National Laboratory Equation of State Database," Technical Report LA-UR-92-3407, Los Alamos National Laboratory, 1992.

[18] Zucker, R.D. \& Biblarz, O., Fundamentals of Gas Dynamics, 2nd ed., Wiley: Hoboken, NJ, pp. 147-158, 2002.

[19] Roache, P.J., Verification and Validation in Computational Science and Engineering, Hermosa Publishers: Albuquerque, NM, pp. 109-131, 2009.

[20] Weitzman, M.S., Measures of overlap of income distributions of white and negro families in the United States. U.S. Government Printing Office, 22, pp. 1-37, 1970.

[21] Clemmons, T.E. \& Bradley, E.L., A nonparametric measure of the overlapping coefficient. Computational Statistics and Data Analysis, 34, pp. 51-61, 2000. 